\title{
Assessing a multidisciplinary survivorship program in a group of predominantly Hispanic women with breast cancer
}

\author{
Zeina A Nahleh, MD, FACP, ${ }^{a}$ Alok Kumar Dwivedi, PhD, ${ }^{b}$ Rosalinda Heydarian, ANP, ${ }^{c}$ \\ Rebecca Pasillas, PhD, ${ }^{\mathrm{d}}$ Danielle Liss, BS, ${ }^{\mathrm{c}}$ Luis Sanchez, BA, ${ }^{\mathrm{c}}$ Cecilia Ochoa, MPH, ${ }^{\mathrm{c}}$ and \\ Safa E Farrag, MD, FACP
}

${ }^{a}$ Department of Hematology Oncology, Maroone Cancer Center, Cleveland Clinic Weston, Florida; ${ }^{b}$ Division of Biostatistics \& Epidemiology (Department of Biomedical Sciences), 'Department of Hematology Oncology, and dDepartment of Psychiatry, Texas Tech University Health Sciences Center, E1 Paso, Texas; and 'Department of Internal Medicine, SSM St Mary's Health Center, Saint Louis, Missouri.

Background The prevalence of long-term mental and physical illness is significant among many breast cancer survivors. We have previously reported that Hispanic survivors of breast cancer have a diminished mental and physical health-related quality of life (QoL), though in general, there is a paucity of data for this population and other minorities. Implementing a cancer survivorship program as an integral component of care during and after treatment could improve long-term QoL in survivors of breast cancer and empower them in the transition from treatment to survivorship.

Objective To determine the value of implementing a multidisciplinary survivorship program that includes psychological counselling and interventions based on mindfulness and counselling.

Methods Survivors of stages I-III breast cancer were recruited within 5 years of their diagnosis at an institution in El Paso, Texas, where the majority of patients are of Hispanic ethnicity, to participate for a year in a multidisciplinary program that provided psychological counselling in conjunction with oncology care, dietary advice, and an 8-week course based on mindfulness-based stress reduction. Self-administered questionnaires - the Patient Health Questionnaire-9 for depression, the General Anxiety Disorder-7, and the Short-Form Health Survey-36 (version 2) for QoL - were completed at baseline and every 3 months for 12 months.

Results 94 patients, of whom $>90 \%$ were Hispanic, were included in this 12 -month analysis. 60 patients (63.8\%) completed all follow-ups at 12 months. Significant improvement from baseline was observed for patient health and anxiety measures for all ethnic groups combined.

Limitations Small, single-center study; no control arm using other possible interventional methods

Conclusion All breast cancer survivors who participated in this 12-month multidisciplinary interventional survivorship program reported less anxiety and depression, compared with baseline measurements and showed a trend toward improved Mental Component Summary of QoL. We believe these findings are representative of outcome in Hispanic women since $>90 \%$ of participants were Hispanic.

Funding Cancer Prevention and Research Institute of Texas (CPRIT)-RP1 20528

$\mathrm{B}$ reast cancer survivors comprise the most prevalent cancer survivor population in the United States. ${ }^{1}$ The number of breast cancer survivors is increasing because of early detection and diagnosis, and advances in treatment have resulted in increased life expectancy. Therefore, greater attention is needed to improve the long-term quality of life of these survivors and to help them re-adjust to normal life. For many women, although the medical treatment may have been completed, the recovery process may have not. ${ }^{2}$ The prevalence of long-term mental and physical illness is significant among many breast cancer survivors. Long-term mental consequences may include memory problems, anxiety, depression, and fear of recurrence ${ }^{3}$, and long-term physical consequences may include pain, fatigue, and lymphedema, among others. ${ }^{4}$

El Paso, Texas, is the fourth most populous city in Texas and has a Hispanic majority. This provides an opportunity to conduct clinical research targeting participants of Hispanic descent. Several studies have noted the influence of race/ethnicity on the psychosocial function of breast cancer survivors. ${ }^{5,6}$ We have previously reported that Hispanic

Accepted for publication January 4, 2017. Correspondence: Safa Farrag, MD, FACP; safa.farrag@ssmhealthx.com. Disclosures: The authors report no disclosures or conflicts of interest. JCSO 2017;15(4):e208-e216. (02017 Frontline Medical Communications. doi: https://doi.org/10.12788/jicso.0322 
breast cancer survivors might experience decreased mental and physical health-related quality of life (QoL) which limit their normal social functioning. ${ }^{6}$ Other studies have similarly reported poor outcomes of breast cancer survivors and higher rates of fatigue and depression among Hispanic patients. ${ }^{7}$ However, there is a paucity of research addressing specific interventions needed to improve these outcomes and provide better QoL for breast cancer survivors. ${ }^{8,9}$ In addition, a few survivorship care interventions have focused on minorities. We sought to assess whether a multidisciplinary cancer survivorship program in a primarily Hispanic populated area would lead to improved QoL and reduce anxiety and depressive symptoms among breast cancer survivors.

\section{Methods}

After obtaining Institutional Review Board approval, we recruited consecutive patients who were treated at our institution during October 2013-October 2014 and obtained informed consent from them. The participants were within the first 5 years after diagnosis with stages I-III breast cancer and had completed surgery, chemotherapy, and/or radiation therapy. We sought to determine whether breast cancer survivors would benefit from this intervention as determined by improvement of performance at 12 months compared with baseline based on the following self-reported validated questionnaires: Patient Health Questionnaire-9 (PHQ-9) for depression; General Anxiety Disorder-7 (GAD-7); and Short-Form Health Survey-36 (SF-36, version 2) for patient quality of life. The participants were enrolled in a comprehensive survivorship program staffed by an oncologist, an oncology nurse practitioner, a nutritionist, and a certified clinical psychologist who had trained in mindfulness-based stress reduction (MBSR).

\section{Interventions}

The participants received a one-on-one individual psychological consultation visit every 3 months for 20-45 minutes during which the psychologist addressed each patient's emotional and psychological issues in depth, discussed relaxation techniques, and provided psychosocial counselling. In addition, all participants were asked to attend an 8-week-course (in Spanish or English) using MBSR, an interventional program in which participants receive training to promote reduction of stress by self-regulating mindfulness practice. ${ }^{3,10}$ Our institution's MBSR program consists of a weekly 2-hour class for 8 sessions or more. The program is provided 3 times a year, in English and Spanish. It includes the following components:

- Learning various mindfulness meditation techniques (eg, body scans, awareness of breathing, sitting/walking meditations);

- Practicing the mindfulness techniques in class; and
- Practicing techniques at home through audiorecordings of mindfulness meditation exercises and daily diary writing.

Participants were provided with a workbook on MBSR in their preferred language. ${ }^{11}$ In addition to the psychological component, they were also provided with oncologic evaluations by an oncology nurse practitioner. The nurse practitioner met with participants every 3 months and provided each one with a personalized summary of all the treatments received and routine oncology follow-up care in consultation with the patients' regular oncologists. This care also addresses the long-term sequelae of treatment, including arthritis and osteoporosis, referrals to receive screening for other cancers (eg, cervical and colon cancer), and genetic counselling as appropriate. In addition, a nutritionist provided general dietary advice in individual and group sessions every 3 months.

The self-administered questionnaires, PHQ-9, GAD7, and SF-36, were completed at baseline, and every 3 months for 12 months. The scores were reviewed by the psychologist and the oncologist. The PHQ-9 was used to initially screen survivors for depression and monitor their improvement after the intervention. The PHQ-9 is a reliable and validated self-administered depression module. ${ }^{12}$ The PQH-9 exclusively focuses on the 9 diagnostic criteria for DSM-IV depression disorder and it can be used as a useful measure for monitoring outcomes of depression therapy. A score of 5-14 suggests mild-moderate depression, and a score of $>15$ suggests severe depression

The survivors were screened for anxiety using the GAD7, a brief 7-item self-report scale to identify probable cases of anxiety disorder that has been shown to be an efficient tool for screening and assessing the severity of anxiety. ${ }^{13}$ For GAD-7, a score of 5 or higher is suggestive of anxiety. Scores of 5, 10, and 15 represent cut-off points for mild, moderate, and severe anxiety, respectively.

Survivor QoL was evaluated using the SF-36 questionnaire, a multipurpose survey containing 36 questions. It ranges from $0-100$ and a score that is $<50.0$ is considered low. The lower the score, the worse the mental or physical function. ${ }^{14}$ The SF-36 yields a patient profile of 8 health domains - vitality, physical functioning, bodily pain, general health perceptions, physical, emotional, and social role functioning; and mental health. ${ }^{15,16}$ A score of 50.0 on either the Physical Component Summary (PCS - vitality, physical functioning, bodily pain, general health perceptions, physical role functioning) or Mental Component Summary (MCS - emotional and social role functioning, and mental health) is consistent with the US norm.

\section{Statistical analysis}

In this study, the primary objective was to use the MBSR survivorship program to improve the survivors' outcomes at 12 months compared with baseline using the following measures: PHQ-9 for depression, GAD-7 for anxiety, and 
TABLE 1 Summary of baseline characteristics of survivors

\begin{tabular}{|c|c|c|c|c|}
\hline \multirow[b]{2}{*}{ Variable } & \multirow[b]{2}{*}{ Overall } & \multicolumn{2}{|c|}{$\begin{array}{l}\text { Survivors completing } \\
12 \text { mo. of follow-up, } n(\%)\end{array}$} & \multirow[b]{2}{*}{$P$-value } \\
\hline & & $\begin{array}{l}\text { Attended } \\
<8 \text { sessions }\end{array}$ & $\begin{array}{l}\text { Completed } \\
\geq 8 \text { sessions }\end{array}$ & \\
\hline Mean age, y (SD) & $54.40(8.71)$ & $54.98(8.58)$ & $54.87(8.14)$ & .96 \\
\hline \multirow[t]{2}{*}{ Mean disease duration, y (SD) } & $2.98(2.19)$ & $3.0(2.45)$ & $2.67(2.02)$ & .82 \\
\hline & $\begin{array}{c}n(\%) \\
(N=94)\end{array}$ & $\begin{array}{c}n(\%) \\
(N=45)\end{array}$ & $\begin{array}{c}n(\%) \\
(N=15)\end{array}$ & \\
\hline Race & & & & .84 \\
\hline Hispanic & $85(90.4)$ & $41(91.11)$ & $14(93.33)$ & - \\
\hline Asian & $3(3.19)$ & $1(2.22)$ & $0(0)$ & - \\
\hline White & $6(6.38)$ & $3(6.67)$ & $1(6.67)$ & - \\
\hline Diagnosis & & & & .27 \\
\hline Ductal & 79 (84.04) & $39(86.67)$ & $12(80)$ & - \\
\hline Lobular & $10(10.64)$ & $4(8.89)$ & $2(13.33)$ & - \\
\hline Ductal and lobular & $3(3.19)$ & $2(4.44)$ & $0(0)$ & - \\
\hline Other & $2(2.13)$ & $0(0)$ & $1(6.67)$ & - \\
\hline Type of chemotherapy & & & & .74 \\
\hline Anthracycline + taxanes & $45(47.87)$ & $24(53.33)$ & $10(66.67)$ & - \\
\hline Anthracycline only & $2(2.13)$ & $2(4.44)$ & $0(0)$ & - \\
\hline Taxanes only & $22(23.4)$ & $7(15.56)$ & $2(13.33)$ & - \\
\hline Other & $1(1.06)$ & 0 & 0 & - \\
\hline None & $24(25.53)$ & $12(26.67)$ & $3(20.00)$ & - \\
\hline Type of hormonal therapy & & & & .42 \\
\hline None & $27(28.72)$ & $13(28.89)$ & $5(33.33)$ & - \\
\hline Tamoxifen & $21(22.34)$ & $11(24.44)$ & $2(13.33)$ & - \\
\hline Aromatase inhibitor & $29(30.85)$ & $14(31.11)$ & $4(26.67)$ & - \\
\hline Other & $1(1.06)$ & $0(0)$ & $1(6.67)$ & - \\
\hline Tamoxifen and anastrazole & $16(17.02)$ & $7(15.56)$ & $3(20.00)$ & - \\
\hline Trastuzumab & & & & .67 \\
\hline No & 78 (82.98) & $38(84.44)$ & $14(93.33)$ & - \\
\hline Yes & $16(17.02)$ & $7(15.56)$ & $1(6.67)$ & - \\
\hline Radiation & & & & .71 \\
\hline No & $19(20.21)$ & $9(20)$ & $2(13.33)$ & - \\
\hline Yes & 75 (79.79) & $36(80)$ & $13(86.67)$ & - \\
\hline Surgery & & & & .57 \\
\hline Lumpectomy & $48(51.06)$ & $25(55.56)$ & $7(46.67)$ & - \\
\hline Mastectomy & $46(48.94)$ & $20(44.44)$ & $8(53.33)$ & - \\
\hline Estrogen receptor & & & & .74 \\
\hline Negative & $27(28.72)$ & $12(26.67)$ & 5 (33.33) & - \\
\hline Positive & $67(71.28)$ & 33 (73.33) & $10(66.67)$ & - \\
\hline
\end{tabular}




\section{TABLE 1 Continued}

\begin{tabular}{|c|c|c|c|c|}
\hline \multirow[b]{2}{*}{ Variable } & \multirow[b]{2}{*}{ Overall } & \multicolumn{2}{|c|}{$\begin{array}{l}\text { Survivors completing } \\
12 \text { mo. of follow-up, } n(\%)\end{array}$} & \multirow[b]{2}{*}{$P$-value } \\
\hline & & $\begin{array}{l}\text { Attended } \\
<8 \text { sessions }\end{array}$ & $\begin{array}{l}\text { Completed } \\
\geq 8 \text { sessions }\end{array}$ & \\
\hline Progesterone receptor & & & & .22 \\
\hline Negative & $39(41.49)$ & 15 (33.33) & $8(53.33)$ & - \\
\hline Positive & $55(58.51)$ & $30(66.67)$ & $7(46.67)$ & - \\
\hline HER2-neu & & & & 1.00 \\
\hline Negative & 75 (79.79) & 37 (82.22) & $13(86.67)$ & - \\
\hline Positive & $19(20.21)$ & $8(17.78)$ & $2(13.33)$ & - \\
\hline Family history of cancer & & & & .49 \\
\hline None or unknown & $51(54.26)$ & $26(57.78)$ & $7(46.67)$ & - \\
\hline First degree family & $30(31.91)$ & $15(33.33)$ & $5(33.33)$ & - \\
\hline Second degree family & $13(13.83)$ & $4(8.89)$ & $3(20.00)$ & - \\
\hline Obesity & & & & 1.00 \\
\hline No & $41(43.62)$ & $20(44.44)$ & $6(40.00)$ & - \\
\hline Yes & $53(56.38)$ & $25(55.56)$ & $9(60.00)$ & - \\
\hline Comorbidities & & & & .20 \\
\hline None & $47(50)$ & $23(51.11)$ & $6(40.00)$ & - \\
\hline Diabetes mellitus & $2(2.13)$ & $1(2.22)$ & $2(13.33)$ & - \\
\hline Hyperlipidemia & $10(37.23)$ & $4(8.89)$ & $3(20.00)$ & - \\
\hline Others & $35(37.23)$ & $17(37.78)$ & $4(26.67)$ & - \\
\hline
\end{tabular}

HER2, human epidermal growth factor receptor 2; MBSR, mindfulnes-based stress reduction

SF-36 for QoL using the PCS and MCS. Quantitative data were described using the mean and standard deviation, and categorical data were described using frequency and percentage. The outcome measures were compared between patients who completed 12-month follow-up and those who did not, using unpaired $t$ test. The change in outcome measures at 12 months from baseline was evaluated using paired $t$ test. The effect of intervention was summarized using relative percentage change. The "dose" of the intervention was categorized the number of MBSR sessions $-\leq 4$ sessions, $5-7$ sessions, or $\leq 8$ sessions. The change in outcome measures were compared among three groups using 1-way analysis of variance (ANOVA) followed by post hoc multiple comparison using the Bonferroni adjustment. In addition, the effect of intervention on each outcome was evaluated by important baseline characteristics of patients. In each subgroup, the changes were compared with baseline measures using the paired $t$ test, whereas changes in outcome between groups were compared using the unpaired $t$ test. Statistical analyses were conducted using SAS 9.3. $P$-values less than $5 \%$ were considered to be significant.

\section{Results}

A total of 94 survivors of breast cancer were included in this study and $60(63.8 \%)$ completed the 12 months of follow-up. The average age of the participants was 54.4 years (SD, 8.7), and $90.4 \%$ were Hispanic (Table 1). Tumor characteristics were as follows: invasive ductal carcinoma (84.04\%), estrogen receptor-positive (ER-, 71.28\%), progesterone receptor-positive (PR-, 58.51\%), and HER2neu-positive (20\%). In regard to therapy received, $48 \%$ of the participants had received anthracycline- and taxanebased adjuvant chemotherapy and $23 \%$, nonanthracyclinebased chemotherapy; $71 \%$ had received anti-estrogen (hormonal) therapy and $80 \%$, radiation therapy. In regard to surgery, half of the participants had a lumpectomy, and half, a mastectomy. The trends in the outcome measures over the follow-up period are show in the Figure 1.

The effect of survivorship program intervention on SF-36 (PCS and MCS), anxiety (GAD-7), and (PHQ-9) at 12 months are shown in Table 2, which also includes the 12-month effects on the body-mass index (BMI). The $P$-values correspond to the comparison of mean change in scores between baseline and 12-month follow-up. 
Significant improvement from baseline was observed for PHQ-9 $(P=.0031)$ and GAD-7 $(P=.0027)$. There was a significant trend toward improvement $(14 \%)$ relative to baseline in the SF-36 MCS at 12 months $(P=.097)$. Although the SF-36 PCS improved numerically, it did not reach to a statistical significance level $(P=.896)$. The BMI at 12 months was found to be statistically significantly

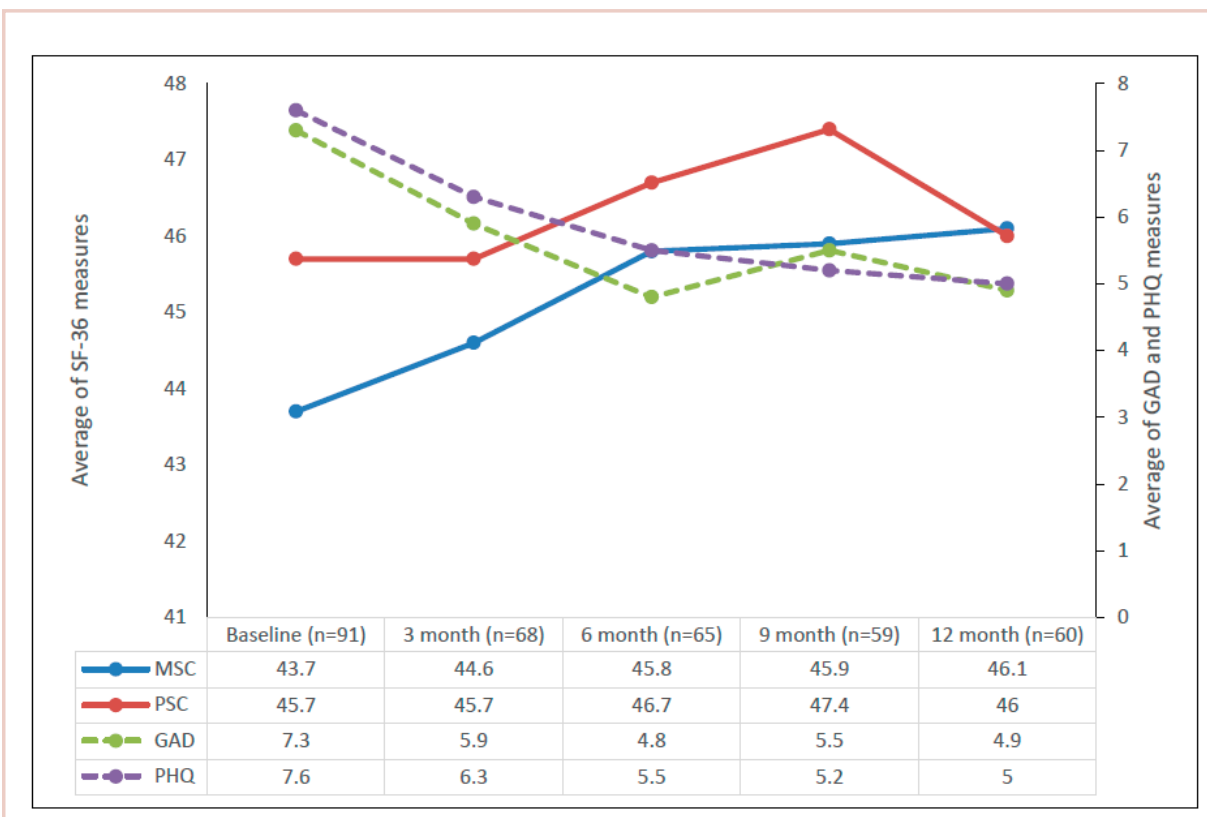

FIGURE 1 Trend in the outcome measures over the follow-up period.

GAD-7, General Anxiety Disorder-7; MCS, Mental Component Summary (vitality, role emotional, social functioning, and mental health); PCS, Physical Component Summary (physical functioning, role physical, bodily pain, and general health dimensions); PHQ-9, Patient Health Questionnaire-9 (for depression); SF-36, Short-Form Health Survey-36 (version 2, for quality of life)

TABLE 2 Effect of multidisciplinary intervention on outcomes at 12 months compared with baseline $(n=60)$

\begin{tabular}{|c|c|c|c|c|c|}
\hline Variable & $\begin{array}{l}\text { Mean base- } \\
\text { line value }\end{array}$ & $\begin{array}{l}\text { Mean } \\
12 \text {-mo } \\
\text { value }\end{array}$ & $\begin{array}{l}\text { Mean \% } \\
\text { change }^{\alpha}\end{array}$ & $\begin{array}{c}\text { Mean } \\
\text { difference } \\
{[95 \% \mathrm{Cl}]}\end{array}$ & $P$-value ${ }^{c}$ \\
\hline MCS & 43.70 & 46.10 & 13.94 & $\begin{array}{c}2.37 \\
{[-0.44-5.19]}\end{array}$ & .0965 \\
\hline PCS & 45.75 & 46.02 & 1.21 & $\begin{array}{c}-0.13 \\
{[-2.06-1.81]}\end{array}$ & .90 \\
\hline GAD-7 & 7.30 & 4.92 & -15.90 & $\begin{array}{c}-2.22 \\
{[-3.63--0.8]}\end{array}$ & .0027 \\
\hline PHQ-9 & 7.62 & 5.00 & -15.89 & $\begin{array}{c}-2.32 \\
{[-3.82--0.81]}\end{array}$ & .0031 \\
\hline $\mathrm{BMI},{ }^{\mathrm{c}} \mathrm{kg} / \mathrm{m}^{2}$ & 31.32 & 32.36 & 2.71 & $\begin{array}{c}0.82 \\
{[0.36-1.28]}\end{array}$ & .0007 \\
\hline
\end{tabular}

BMI, body-mass index; GAD-7, General Anxiety Disorder-7; MCS, Mental Component Summary; PCS, Physical Component Summary; PHQ-9, Patient Health Questionnaire-9 for depression

a Mean percent change is defined as mean of (12-month value minus baseline value)/baseline value. ${ }^{b}$ Mean difference is defined as mean of (12-month value minus baseline value). ${ }^{c} P$-value reflects comparison of mean change in values. 'Obesity range, $>30 \mathrm{~kg} / \mathrm{m}^{2}$. increased compared with baseline $(P=.0007)$.

The effect of the number of MBSR sessions attended on the outcome measures is summarized in Table 3. There were significant improvements in the 12-month MCS scores for patients who completed 5-7 sessions of MBSR or $\geq 8$ sessions, compared with patients who completed $\leq 4$ sessions of MBSR. There was an improvement observed in PCS scores only among patients who received at least 8 sessions of MBSR. There was a marked improvement observed in GAD-7 and PHQ-9 among patients who received $\geq 8$ sessions. There was no statistically significant change in the GAD-7 or PHQ-9 scores between patients who received $\leq 4$ sessions and $5-7$ sessions. No significant association was obtained between number of MBSR sessions attended and BMI.

The effect of survivorship program intervention on all outcomes according to important baseline cofactors is shown in Table 4. As such, there were no significant differences in changes in the outcome measures after intervention according to any considered baseline characteristics. However, the effect of survivorship program intervention was more pronounced in patients who were $\geq 3$ years away from their initial diagnosis and who had attended a minimum of $80 \%$ of the 3-monthly visits and received a minimum of $8 \mathrm{MBSR}$ sessions.

The mean baseline PCS and MCS scores of the SF-36 were 43.7 and 45.8, respectively, indicating that the participants' scores were significantly less than half the standard deviation below the US norm (50.0; SD, 10). The SF-36 health-related QoL categories showed that, on an average, scores improved by more than 4 units for emotional and physical role functions, vitality, and mental health compared with baseline. In addition, scores improved by about 2 units for general health and social functioning compared with baseline data. In all, $65 \%$ of survivors had difficulty preforming work at baseline, but that dropped to $55 \%$ after enrollment in the program; and $60 \%$ had originally reduced the amount of time spent on work, but that increased to $50 \%$ 
after the intervention. Also of note is that $70 \%$ of survivors reported accomplishing less than they would like to have (role physical) before the intervention, but that was reduced to $57 \%$ after the intervention. Similarly, $77 \%$ of survivors felt worn out at baseline, compared with $65 \%$ at the 12 -month follow-up; and $88 \%$ felt tired at baseline, but that percentage was reduced to $68 \%$ after the intervention. Before the intervention, $60 \%$ of the participants reported that they had been very nervous, and $45 \%$ said they had been so down in the dumps that nothing could cheer them up, but those percentages were reduced to $43 \%$ and $32 \%$, respectively, after intervention. Before intervention, $63 \%$ of the women said they felt depressed and that was reduced to $50 \%$ after the intervention.

\section{Discussion}

In this study, we showed that a group of predominately Hispanic breast cancer survivors benefited from participating in a multidisciplinary cancer survivorship program that emphasized in-depth psychological care and MBSR. They also benefited from an education effort that included providing survivors with personalized summaries of their treatment and oncology survivorship care, addressing potential long-term side effects of treatment, referral for genetic counselling and screening for other cancers as appropriate, as well dietary advice. We found significant improvement compared with baseline in both mental and physical determinants of the patient-reported outcomes, including anxiety (GAD-7), depression (PHQ-9), and HR-QoL (PCS) and (MCS). Survivors demonstrated significant improvement on the MCS and PHQ-9 if they attended 5 or more sessions of the 8-week MBSR course, and attending 8 sessions was associated with significant improvement in GAD-7 and PCS. This might suggest that survivors who are more motivated do benefit the most from such program.

To our knowledge, this study is the first to address the benefit of the MBSR intervention in Hispanic breast cancer survivors. In a randomized controlled trial that included breast cancer survivors with stages 0 -III breast cancer who completed surgery, adjunctive radiation, and/or chemotherapy, MBSR was shown to reduce the symptoms of depression and anxiety and increase energy and physical functioning compared with participants who received "usual care". ${ }^{3}$ Furthermore, Bower and colleagues have reported improvements in sleep, fatigue, and pro-inflammatory signaling in younger survivors of breast cancer. ${ }^{17} \mathrm{~A}$ similar standardized MBSR program was tested on Danish women who had been treated for stage I-III breast cancer ${ }^{18}$ and the results showed reduced levels of anxiety and depression at the 12-month follow-up. A similar study by Hoffman and colleagues $^{19}$ reported improved mood, breast- and endocrine-related quality of life, and well-being with MBSR compared with standard care in women with stage 0-III breast cancer.

Several theories have been suggested to explain how MBSR reduces symptoms of depression, anxiety, and fear of recurrence in breast cancer survivors, one of which is that it provides supportive interaction between group members to practice meditation and apply mindfulness in daily situations. ${ }^{3}$ In addition, evidence is beginning to emerge that stress-reducing interventions such as MBSR may improve telomere length (TL) and telomerase activity (TA), the markers for cellular aging, psychological stress, and disease risk. ${ }^{20-24}$ Lengacher and colleagues conducted a randomized controlled study to investigate the effects of MBSR on TL and TA in women with breast cancer, and suggested that MBSR increases telomere length and telomerase activity. ${ }^{25}$ The 142 patients with stages 0 -III breast cancer had completed adjuvant treatment with radiation and/ or chemotherapy at least 2 weeks before enrollment and within 2 years of completion of treatment with lumpectomy and/or mastectomy. They were randomly assigned to either a 6-week MBSR for breast cancer program or usual care. ${ }^{25}$ Assessments of TA and TL were obtained along with psychological measurements at baseline, 6 weeks, and 12 weeks after the patients had completed the MBSR program. The mean age of the participants was 55.3 years; $72 \%$ were non-Hispanic white; $78 \%$ had stage I or II cancer; and 36\% received both chemotherapy and radiation. In analyses adjusted for baseline TA and psychological status, TA increased steadily by about $17 \%$ over 12 weeks in the MBSR group, compared with about $3 \%(P<.01)$ in
TABLE 3 Effect of number of MBSR sessions attended on outcomes at 12 months compared with baseline $(\mathrm{n}=60)$

\begin{tabular}{|c|c|c|c|c|}
\hline \multirow[b]{2}{*}{ Variable } & \multicolumn{3}{|c|}{ Mean difference in score [12 mo - baseline] (SD) } & \multirow[b]{2}{*}{ P-value } \\
\hline & $\begin{array}{c}\leq 4 \text { sessions } \\
(n=26)\end{array}$ & $\begin{array}{c}\text { 5-7 sessions } \\
(\mathrm{n}=19)\end{array}$ & $\begin{array}{c}\geq 8 \text { sessions } \\
(n=15)\end{array}$ & \\
\hline MCS & $-1.2(9.1)$ & $3.0(10.1)$ & $7.8(12.8)^{*}$ & .0359 \\
\hline PCS & $-0.3(6.7)$ & $-1.2(8.1)$ & $1.5(8.1)$ & .58 \\
\hline GAD-7 & $-1.6(5.2)$ & $-0.8(4.6)$ & $-5.0(6.2)^{*}$ & .0652 \\
\hline PHQ-9 & $-0.5(5.2)$ & $-1.4(3.8)$ & $-6.7(6.9) * \dagger$ & $.0022^{*} \dagger$ \\
\hline $\mathrm{BMI},{ }^{a} \mathrm{~kg} / \mathrm{m}^{2}$ & $1.1(1.9)$ & $0.4(1.7)$ & $0.9(1.6)$ & .46 \\
\hline
\end{tabular}

BMI, body mass index; GAD-7, General Anxiety Disorder-7; MBSR, Mindfulness-based stress reduction; MCS, Mental Component Summary; PCS, Physical Component Summary; PHQ-9, Patient Health Questionnaire-9 for depression 'Obesity range, $>30 \mathrm{~kg} / \mathrm{m}^{2}$.

*Significant difference between $\leq 4$ and $\geq 8$ sessions. $†$ Significant difference between $5-7$ and $\geq 8$ sessions. 
the control group. No difference was observed for TL $(P=$ .92). The authors concluded that the data provide preliminary evidence that MBSR increases TA in peripheral blood mononuclear cells from breast cancer patients and have implications for understanding how MBSR may extend cell longevity at the cellular level.

In another study among healthy volunteers who were randomly assigned to a 3-month meditation retreat or a control group, the 30 participants in the meditation group had higher TA compared with controls. ${ }^{20}$ In a nonrandomized study among prostate cancer patients, TA increased and psychological stress decreased following a stress-reducing, lifestyle-modification program. ${ }^{21}$ The results of another intervention study among overweight women showed

TABLE 4 Effect of intervention on outcomes by different characteristics of participants

\begin{tabular}{|c|c|c|c|c|c|}
\hline \multirow[b]{2}{*}{ Variable } & \multicolumn{2}{|c|}{ Baseline group } & \multicolumn{2}{|c|}{ Comparator group } & \multirow[b]{2}{*}{$P$-value** } \\
\hline & Change (SD) & P-value* & Change (SD) & $P$-value* & \\
\hline Time from diagnosis & \multicolumn{2}{|c|}{$<3$ y $(n=36)$} & \multicolumn{2}{|c|}{$\geq 3$ y $(n=24)$} & \\
\hline MCS & $0.77(9.26)$ & .62 & 4.77 (12.79) & .08 & .17 \\
\hline PCS & $-1.1(7.86)$ & .41 & $1.33(6.8)$ & .34 & .22 \\
\hline GAD-7 & $-1.31(3.93)$ & .05 & $-3.58(7.08)$ & .021 & .17 \\
\hline PHQ-9 & $-1.44(5.2)$ & .10 & $-3.63(6.53)$ & .012 & .16 \\
\hline $\mathrm{BMI}, \mathrm{kg} / \mathrm{m}^{2}$ & 1.03 (1.99) & .004 & $0.51(1.37)$ & .08 & .27 \\
\hline Chemotherapy & \multicolumn{2}{|c|}{ No anthracyclines $(n=15)$} & \multicolumn{2}{|c|}{ Anthracyclines ( $n=45)$} & \\
\hline MCS & $4.04(16.21)$ & .35 & $1.82(8.61)$ & .16 & .62 \\
\hline PCS & 1.01 (9.59) & .69 & $-0.51(6.74)$ & .62 & .50 \\
\hline GAD-7 & $-4(6.41)$ & .03 & $-1.62(5.06)$ & .037 & .15 \\
\hline PHQ-9 & $-0.73(5.84)$ & .63 & $-2.84(5.78)$ & .002 & .23 \\
\hline $\mathrm{BMI}, \mathrm{kg} / \mathrm{m}^{2}$ & $0.38(1.88)$ & .45 & $0.97(1.73)$ & .001 & .27 \\
\hline Obesity & \multicolumn{2}{|c|}{ No obesity $(n=26)$} & \multicolumn{2}{|c|}{ Obesity (n = 34) } & \\
\hline MCS & 2.01 (5.99) & .10 & 2.65 (13.59) & .25 & .82 \\
\hline PCS & $-0.74(5.59)$ & .51 & $0.34(8.72)$ & .82 & .56 \\
\hline GAD-7 & $-1.58(3.71)$ & .04 & $-2.71(6.53)$ & .021 & .40 \\
\hline PHQ-9 & $-2.92(4.44)$ & .003 & $-1.85(6.71)$ & .12 & .46 \\
\hline $\mathrm{BMI}, \mathrm{kg} / \mathrm{m}^{2}$ & $0.96(1.47)$ & .003 & 0.71 (1.99) & .044 & .60 \\
\hline Any comorbidity & \multicolumn{2}{|c|}{ No comorbidity $(n=29)$} & \multicolumn{2}{|c|}{ Comorbidity $(n=31)$} & \\
\hline MCS & $1.92(10.6)$ & .34 & $2.80(11.31)$ & .18 & .76 \\
\hline PCS & $-1.08(7.36)$ & .44 & $0.77(7.62)$ & .58 & .34 \\
\hline GAD-7 & $-2.48(5.43)$ & .020 & $-1.97(5.59)$ & .060 & .72 \\
\hline PHQ-9 & $-2.66(5.58)$ & .016 & $-2.00(6.1)$ & .078 & .67 \\
\hline $\mathrm{BMI}, \mathrm{kg} / \mathrm{m}^{2}$ & $0.71(1.58)$ & .023 & $0.93(1.96)$ & .013 & .63 \\
\hline No. of MBSR sessions & \multicolumn{2}{|c|}{ MBSR, $<8$ sessions $(n=45)$} & \multicolumn{2}{|c|}{ MBSR, $\geq 8$ sessions $(n=15)$} & \\
\hline MCS & $0.58(9.69)$ & .6893 & 7.75 (12.77) & .034 & .0259 \\
\hline PCS & $-0.68(7.28)$ & .5365 & $1.52(8.12)$ & .4803 & .3296 \\
\hline GAD-7 & $-1.29(4.93)$ & .0864 & $-5(6.23)$ & .0077 & .0217 \\
\hline PHQ-9 & $-0.87(4.63)$ & .2163 & $-6.67(6.92)$ & .0022 & .0071 \\
\hline $\mathrm{BMI},{ }^{a} \mathrm{~kg} / \mathrm{m}^{2}$ & $0.8(1.85)$ & .0058 & $0.88(1.57)$ & .0489 & .8782 \\
\hline
\end{tabular}

BMI, body mass index; GAD-7, General Anxiety Disorder-7; MBSR, Mindfulness-based stress reduction, MCS, Mental Component Summary; PCS, Physical Component Summary; PHQ-9, Patient Health Questionnaire-9 for depression

abesity range, $>30 \mathrm{~kg} / \mathrm{m}^{2}$.

*P-value within group. ${ }^{*} P$-value between groups. 
improvement in distress, eating behavior, and metabolic health in women participating in a MBSR program, all of which correlated with increases in TA. ${ }^{22}$ Most recently, researchers explored the impact on TA of a Kirtan Kriya yogic meditation intervention compared with exposure to relaxing music in 39 dementia family caregivers. The yogicmeditation intervention group had a $43 \%$ increase in TA after the 8-week intervention period compared with $3.7 \%$ the music group $(P<.05) .{ }^{23}$ Finally, among 22 patients with cervical cancer who were randomized to a psychosocial telephone counseling intervention, ${ }^{24}$ investigators found a significant association between increased TL and changes in psychological distress. ${ }^{20}$ Findings from other studies have assessed interventions to improve outcome of breast cancer survivors, such as the Taking CHARGE self-management intervention that is designed to facilitate the transition to survivorship after breast cancer treatment. ${ }^{8}$ Another intervention using home-based physical activity was shown in a randomized controlled trial to improve self-reported physical activity, body-mass index, and health-related QoL. ${ }^{9}$ Findings from another study suggested that a combined exercise and psychological counselling program might improve QoL more than a single entity intervention. ${ }^{26} \mathrm{As}$ noted previously, these studies did not focus on minority breast cancer survivors' population, and it is not clear if they are generalizable to Hispanics.

In addition to the MBSR component, our program has also included one-on-one psychological assessment for long-term treatment complications and provided participants with appropriate care and follow-up plans, adding the benefits of self-awareness and self-attention for the survi- vors, which can effectively reduce the fear of recurrence. ${ }^{3}$ Furthermore, we included dietary consults based on general cancer survivor guidelines recommending a high fruit and vegetable diet that is low in fat and sugar. ${ }^{27}$ Healthier dietary lifestyle has been reported to improve breast cancer prognosis, metabolic disease, and cardiovascular outcomes among Hispanic breast cancer survivors. ${ }^{28}$

Our study has some limitations, including a relatively small sample size. It did not include an exercise program, which would have been helpful in addressing the issue of overweight and obesity we encountered in the most of the Hispanic breast cancer survivors (baseline average BMI, $31.32 \mathrm{~kg} / \mathrm{m}^{2}$; obesity range, $>30 \mathrm{~kg} / \mathrm{m}^{2}$ ). Because of the small sample size and nonrandomized design of the study, it is hard to evaluate the confounding effect of time on intervention effect. However, a subgroup analysis by MBSR number of sessions showed that the survivors who completed the full course of MBSR sessions (8 sessions) achieved superior benefit, compared with those who did not complete the full course, which indicates that the intervention did weigh in regardless of time. Despite these limitations, the participants in this interventional program showed improved outcomes, including less anxiety and depression and improved MCS score of the SF-36. A larger and longer follow-up prospective, randomized study is needed to validate the findings of this study. Implementing cancer survivorship as an integral component of cancer care during and after treatment is essential to improve the quality of life of cancer survivors and empower them in their transition from cancer treatment to survivorship.

Randomised controlled trial of a home-based physical activity intervention in breast cancer survivors. https://bmccancer.biomedcentral. com/articles/10.1186/s12885-016-2258-5. Published 2016. Accessed July 25, 2017.

10. Huang J, Shi L. The effectiveness of mindfulness-based stress reduction (MBSR) for survivors of breast cancer: study protocol for a randomized controlled trial. Trials. 2016;17(1):209.

11. Stahl B and Goldstein E, A mindfulness-based stress reduction workbook. 2010: New Harbinger Publications.

12. Kroenke K, Spitzer RL, Williams JB. The PHQ-9: validity of a brief depression severity measure. J Gen Intern Med. 2001;16(9):606-613.

13. Spitzer RL, Kroenke K, Williams JB, Löwe B. A brief measure for assessing generalized anxiety disorder: the GAD-7. Arch Intern Med. 2006;166(10):1092-1097.

14. Ware JE, Kosinski M, Bayliss MS, McHorney CA, Rogers WH, Raczek A. Comparison of methods for the scoring and statistical analysis of SF-36 health profile and summary measures: summary of results from the Medical Outcomes Study. Med Care. 1995;33(4 Suppl):AS264-279.

15. Gandek B, Sinclair SJ, Kosinski M, Ware JE Jr. Psychometric evaluation of the SF-36 health survey in Medicare managed care. Health Care Financ Rev. 2004;25(4):5-25.

16. Ruta D, Garratt A, Abdalla M, Buckingham K, Russell I. The SF-36 health survey questionnaire. A valid measure of health status. BMJ. 1993;307(6901):448-449.

17. Bower JE, Crosswell AD, Stanton AL, et al. Mindfulness meditation for younger breast cancer survivors: a randomized controlled trial. 


\section{Original Report}

Cancer. 2015;121(8):1231-1240.

18. Würtzen H, Dalton SO, Elsass P, et al. Mindfulness significantly reduces self-reported levels of anxiety and depression: results of a randomised controlled trial among 336 Danish women treated for stage I-III breast cancer. Eur J Cancer. 2013;49(6):1365-1373.

19. Hoffman CJ, Ersser SJ, Hopkinson JB, Nicholls PG, Harrington JE, Thomas PW. Effectiveness of mindfulness-based stress reduction in mood, breast- and endocrine-related quality of life, and well-being in stage 0 to III breast cancer: a randomized, controlled trial. J Clin Oncol. 2012;30(12):1335-1342.

20. Jacobs TL, Epel ES, Lin J, et al. Intensive meditation training, immune cell telomerase activity, and psychological mediators. Psychoneuroendocrinology. 2011;36(5):664-681.

21. Ornish D, Lin J, Daubenmier J, et al. Increased telomerase activity and comprehensive lifestyle changes: a pilot study. Lancet Oncol. 2008;9(11):1048-1057.

22. Daubenmier J, Lin J, Blackburn E, et al. Changes in stress, eating, and metabolic factors are related to changes in telomerase activity in a randomized mindfulness intervention pilot study. Psychoneuroendocrinology. 2012;37(7):917-928.

23. Lavretsky H, Epel ES, Siddarth P, et al. A pilot study of yogic meditation for family dementia caregivers with depressive symptoms: effects on mental health, cognition, and telomerase activity. Int J Geriatr Psychiatry. 2013;28(1):57-65.

24. Biegler KA, Anderson AK, Wenzel LB, Osann K, Nelson EL. Longitudinal change in telomere length and the chronic stress response in a randomized pilot biobehavioral clinical study: implications for cancer prevention. Cancer Prev Res (Phila). 2012;5(10):1173-1182.

25. Lengacher CA, Reich RR, Kip KE. Influence of mindfulness-based stress reduction (MBSR) on telomerase activity in women with breast cancer (BC). Biol Res Nurs. 2014;16(4):438-447.

26. Naumann F, Martin E, Philpott M, Smith C, Groff D, Battaglini C. Can counseling add value to an exercise intervention for improving quality of life in breast cancer survivors? A feasibility study. J Support Oncol. 2012;10(5):188-194.

27. Kushi LH, Doyle C, McCullough M, et al. American Cancer Society Guidelines on nutrition and physical activity for cancer prevention: reducing the risk of cancer with healthy food choices and physical activity. CA Cancer J Clin. 2012;62(1):30-67.

28. Greenlee H, Gaffney AO, Aycinena AC, et al. Cocinar para su salud!: randomized controlled trial of a culturally based dietary intervention among Hispanic breast cancer survivors. J Acad Nutr Diet. 2015;115(5):709-723.e3. 